\title{
Adaptación y validación de la versión chilena de la escala de impacto de evento-revisada (EIE-R)
}

\author{
LILIANA CAAMAÑO W. ${ }^{1, a}$, DIEGO FUENTES M. ${ }^{2, a,}$ \\ LUIS GONZÁLEZ B.,3,a, ROBERTO MELIPILLÁN A.,a, \\ MARCELO SEPÚLVEDA C. ${ }^{5, a}$, ELIZABETH VALENZUELA G. ${ }^{6, a}$
}

\section{Assessment of the impact of event scale revised for post traumatic stress disorder in Chilean subjects}

Background: Impact of Event Scale Revised (IES-R), that evaluates the subjective perception of stress, is used to assess post traumatic stress disorder simptoms. Aim: To adapt and validate IES-R to the Chilean population. Material and Methods: Two hundred seventy eight subjects exposed to stressful life events of varying magnitude were assessed using the IES-R, the Beck Depression Inventory, the anxiety and somatoform subscales of the Depressive, Anxiety and Somatoform Disorders Scale and the AUDIT Questionnaire. Results: IES-R had adequate psychometric properties in terms of internal consistency, test-retest reliability, convergent and discriminating validity. It identified a principal factor, explaining $67 \%$ of the variance. Conclusions: The IES-R can be used in the Chilean population to assess the degree of suffering produced by a traumatic event.

(Rev Med Chile 2011; 139: 1163-1168).

Key words: Earthquakes; Psychological tests; Stress disorders.

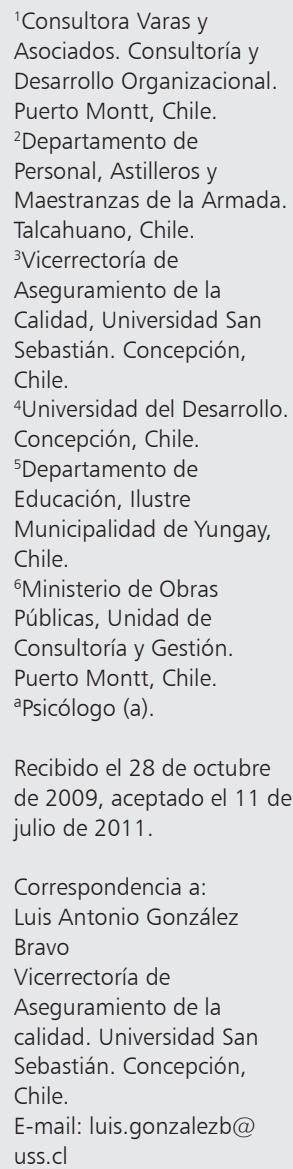

E 1 trastorno por estrés post-traumático (TEPT) es un trastorno que surge como respuesta tardía o diferida a un acontecimiento estresante o a una situación (breve o duradera) de naturaleza excepcionalmente amenazante o catastrófica. Las características típicas del trastorno por estrés post-traumático son episodios reiterados de volver a vivenciar el trauma en forma de reviviscencias o sueños que tienen lugar sobre un fondo persistente de una sensación de "entumecimiento" y embotamiento emocional, de desapego de los demás, de falta de capacidad de respuesta al medio, de anhedonia y de evitación de actividades y situaciones evocadoras del trauma. Suelen temerse y evitarse las situaciones que recuerdan el trauma ${ }^{1}$.

Los criterios mencionados en el DSM-IV TR incluyen, entre otros, la exposición a un acontecimiento traumático, la subsecuente respuesta de temor, la desesperanza u horror intensos, la reexperimentación psíquica del acontecimiento, malestar psicológico y/o respuestas fisiológicas intensas al exponerse a estímulos que recuerdan el evento, evitación de estímulos asociados al trauma, síntomas de aumento de la activación (hiperactivación), etc.

Asimismo, se cuenta desde 1987 con cifras de prevalencia del TEPT en población general mundial, con valores que oscilan entre $0,5 \%$ y $10,8 \%$ para hombres y entre $1,3 \%$ y $18,3 \%$ para mujeres ${ }^{1}$.

La evaluación y diagnóstico del TEPT requiere de instrumentos válidos y confiables, que permitan discriminar la sintomatología propia del trastorno de la sintomatología de otros trastornos comórbidos. Las investigaciones realizadas en torno a la evaluación del TEPT, se han centrado en desarrollar escalas que evalúen tanto la historia 
del trauma relacionada con el evento traumático específico (criterio A, DSM-IV), como también la historia de la sintomatología traumática (criterios B-D, DSM-IV).

Dentro de las escalas más ampliamente utilizadas para evaluar el criterio A del DSM IV con propósitos de tamizaje se pueden mencionar el Inventario de Estrés Traumático (Traumatic Stress Schedule) $)^{2}$, la Lista de Verificación de Estresores Vitales (Life Stressor Checklist-Revised) ${ }^{3}$, y el Cuestionario de Eventos Traumáticos (Traumatic Life Events Questionnaire) ${ }^{4}$.

Por otro lado, en el grupo de aquellas que están orientadas a diagnosticar el TEPT, se pueden nombrar la Escala de Trauma de Davidson ( $D a$ vidson Trauma Scale $)^{5}$ y la Lista de Verificación de Estrés Post-Traumático Uso Civil (PTSD Checklist, Civilian Version) ${ }^{6}$ y en forma más general, el Inventario de Síntomas de Trauma (Trauma Symptom Inventory $)^{7}$. Finalmente, la que es objeto de este estudio de validación, la Escala de Impacto de Evento Revisada, EIE-R ${ }^{8}$.

La escala EIE (IES, Impact Event Scale) fue creada originalmente por Horowitz, Wilner y Alvarez, en $1979^{9}$, y mide el grado de sufrimiento provocado por un evento vital cualquiera, lo que es conceptualizado como estrés subjetivo. Está compuesta de 15 ítems y por dos subescalas: intrusión de pensamientos y evitación. Weiss y Marmar realizaron en el año 1997 una adaptación del instrumento a partir de inclusión de criterios diagnósticos del DSM-IV, incorporando 7 nuevos ítems, evaluando Hiperactivación. Se cuenta para España, con una adaptación realizada por Báguena et al, en el 2001, la cual sirvió de base para el presente estudio ${ }^{10}$.

Variadas investigaciones ${ }^{11,12}$ han utilizado la EIE-R en la evaluación de la sintomatología asociada al TEPT, dando cuenta de su poder discriminante en relación a los trastornos con los cuales presenta comorbilidad, y que avalan su ventaja comparativa con respecto a otras escalas de la misma índole.

\section{Material y Métodos}

\section{Objetivos especificos}

a) Adaptar la versión española de la EIE-R de Báguena et al (2001), de modo que resulte comprensible para la población chilena. b) Establecer las propiedades psicométricas de la EIE-R en una muestra de sujetos chilenos.

\section{Instrumentos}

Tanto el método de validación y adaptación de la EIE-R, como la elección de los instrumentos que a continuación se mencionan, se basaron en el precedente de estudios análogos realizados en otros países para la validación del instrumento.

Los instrumentos utilizados fueron los siguientes:

1. Cuestionario sociodemográfico.

2. Escala de Impacto de Evento-Revisada ${ }^{13}$. Cuenta con 22 ítemes y 3 subescalas: Intrusión, Evitación e Hiperactivación. Utiliza una escala tipo Likert, para la evaluación de la intensidad de la sintomatología $(0,1,2,3,4$. Desde "Nada" hasta "Extremadamente"). Consistencia interna: Intrusión Alfa $=0,89$, Evitación Alfa $=0,84$, Hiperactivación Alfa $=0,82$, Capacidad discriminativa ítem/subescala: Intrusión, $\mathrm{r}$ $=0,52$; Evitación $r=0,40$; Hiperactivación $r$ $=0,55$. Intercorrelaciones por subescalas: $\mathrm{I} / \mathrm{E}$ $\mathrm{r}=0,71 ; \mathrm{I} / \mathrm{H} \mathrm{r}=0,81 ; \mathrm{E} / \mathrm{H} \mathrm{r}=0,65$. Las instrucciones señalan "La siguiente es una lista de dificultades que a veces tienen las personas después de haber vivido un evento estresante. Por favor lea cada frase y luego indique cuán molesto (a) ha sido cada dificultad durante estos ÚLTIMOS SIETE DÍAS, encerrando en un círculo el número de la alternativa que mejor refleje su forma de sentir. ¿Cuánto le molestaron y afligieron estas dificultades?". Se debe señalar que para los fines de esta investigación se utilizará para la primera subescala la traducción original intrusión utilizada por Báguena et al (2001). Previa aplicación piloto, se llevaron a cabo los siguientes análisis para analizar las propiedades psicométricas del instrumento: análisis de consistencia interna, análisis de validez convergente con pruebas criterio, análisis de validez discriminante, análisis de estabilidad temporal y para terminar, análisis factorial exploratorio.

3. Inventario de Depresión de Beck: Beck Depression Inventory, $B D I^{14}$. Confiabilidad: Alfa = 0,83; Presente investigación, Alfa =0,93.

4. Escala para medir trastornos depresivos, por ansiedad y somatomorfos, Subescala de Ansiedad ${ }^{9}$. Confiabilidad: Alfa $=0,92$; Presente investigación, $A l f a=0,86$. 
5. Escala para medir trastornos depresivos, por ansiedad y somatomorfos, Subescala de Trastornos Somatomorfos ${ }^{15}$. Confiabilidad: Alfa $=$ 0,87; Presente investigación, Alfa $=0,84$.

6. Test de identificación de los trastornos debidos al consumo de alcohol, AUDIT ${ }^{16}$. Confiabilidad Test-retest $\mathrm{r}=0,86$; Presente investigación, Alfa $=0,81$.

\section{Muestra}

Muestra no probabilística intencionada. Contestaron el set de instrumentos, previo consentimiento, 278 participantes chilenos entre 16 y 64 años, con un promedio de 36,33 años $(\mathrm{DE}=10,35)$. De ellos, 46,4\% $(\mathrm{n}=129)$ eran hombres y 53,6\% $(n=149)$, eran mujeres. Entre ellos estaban: 1) personas con familiar fallecido en la tragedia de Antuco, en mayo de 2005 ( $n=47$ ); 2) personal asistencial del grupo de operaciones policiales especiales de Carabineros $(n=17) y$ de las Fuerzas Armadas $(\mathrm{n}=16)$, quienes participaron en las labores de rescate y contención de las víctimas de Antuco; 3 ) personas afectadas por las inundaciones de julio de 2006 en Concepción $(\mathrm{n}=23)$; y 4) personas que informaron una discusión de importancia durante los últimos seis meses $(n=175)$. En relación a la escolaridad, los 278 participantes que compusieron la muestra tenían estudios que van desde la Enseñanza Básica incompleta hasta estudios de Postítulo completo: Básica incompleta, 3,6\%; Básica completa, 3,2\%; Media incompleta, 4\%; Media completa, 23\%, Técnico Superior incompleto, 7,6\%; Técnico Superior completo, 19,4\%; Universitario incompleto, 8,3\%; Universitario completo, 25,2\%; Postítulo incompleto, $1,8 \%$, y finalmente, Postítulo completo, $4 \%$.

\section{Resultados}

Inicialmente se realizó una aplicación piloto a una muestra de 50 apoderados del colegio
Etchegoyen de Talcahuano, no encontrándose dificultades mayores en la comprensión global de la EIE-R. No obstante lo anterior, se hicieron modificaciones menores en la pregunta 12 donde se eliminó "...pero no me enfrentaba a ellos", la pregunta 14, donde la expresión "suceso" se cambió por "evento", y la pregunta 19, donde "latidos cardiacos" se cambió por "taquicardia", y "sudoración” por "transpiración”. Todo esto a partir de observaciones de los sujetos relativas a mejorar aun más la claridad en la formulación de dichos ítems.

Una vez realizado este procedimiento, se llevó a cabo la aplicación a la muestra definitiva, obteniéndose los resultados que se expondrán a continuación.

\section{Consistencia interna}

Esta fue adecuada, alcanzando un Alpha de Cronbach de 0,98. Asimismo, las correlaciones entre las subescalas de la EIE-R fueron altas y significativas, tal como se puede observar en la Tabla 1.

\section{Validez convergente}

Para evaluar la validez convergente de la versión chilena de la EIE-R, se calcularon correlaciones de Pearson entre los puntajes obtenidos en la escala y los puntajes obtenidos en la escala de Depresión de Beck $^{14}$, los puntajes obtenidos en las subescalas de ansiedad y de trastornos somatomorfos de la escala para medir trastornos depresivos, por ansiedad y somatomorfos ${ }^{15}$ y la escala AUDIT ${ }^{16}$.

La relación entre estrés subjetivo, evaluada a través de la EIE-R, y la variable depresión muestra un $\mathrm{r}(276)=0,74, \mathrm{p}<0,001(\mathrm{r} 2=0,55)$. La relación entre la variable estrés subjetivo y la variable ansiedad corresponde a un $\mathrm{r}(276)=0,71, \mathrm{p}<0,001$ $(\mathrm{r} 2=0,50)$. La relación entre la variable estrés subjetivo y la variable trastornos somatomorfos muestra un $\mathrm{r}=0,67, \mathrm{p}<0,001(\mathrm{r} 2=0,45)$. Por último, la correlación entre la variable estrés subje-

Tabla 1. Correlaciones entre las sub-escalas de Evitación, Intrusión e Hiperactivación de la EIE-R

\begin{tabular}{|lccc|}
\hline & 1. Evitación & 2. Intrusión & 3. Hiperactivación \\
\hline 1. Evitación & 1 & & \\
2. Intrusión & $0,903^{* *}$ & 1 & 1 \\
\hline 3. Hiperactivación & $0,879^{* *}$ & $0,935^{* *}$ & \\
\hline
\end{tabular}

**Correlaciones significativas al 0,01 (2 colas; $p<0,01$ ). 
tivo y el abuso de alcohol indica un $\mathrm{r}(276)=0,10$, $p>0,005$. Las relaciones son altamente significativas entre estrés subjetivo, depresión, ansiedad y trastornos somatomorfos, y además robustas. Sin embargo, no se observa relación entre el estrés subjetivo y el abuso de alcohol, pudiéndose observar todo esto en la Tabla 2.

\section{Validez discriminante}

En cuanto a la validez de criterio discriminante, se dividió la muestra en tres grupos según la magnitud del estresor que experimentaron: conflicto interpersonal (baja intensidad), inundaciones (mediana intensidad) y muerte de familiares en la tragedia de Antuco (alta intensidad), y se compararon los puntajes obtenidos. Así, mediante un análisis de varianza de un factor (ANOVA), se obtuvo un $\mathrm{F}(2,275)=88,93$, $\mathrm{p}<0,001$. El grupo/ estresor de alta intensidad, obtuvo un puntaje promedio en la EIE - R de 51,81 ( DE = 19,70); el grupo/estresor de mediana intensidad obtuvo un promedio de $27,34(\mathrm{DE}=22,27)$ y por último, el grupo/estresor de baja intensidad obtuvo un promedio de 15,30 ( $\mathrm{DE}=15,72)$. Existen diferencias significativas entre los tipos de muestra en relación a los puntajes obtenidos en la EIE-R. Para complementar este análisis se utilizó la prueba HSD (Diferencia Honestamente Significativa) de Tukey, la cual permitió respaldar las diferencias significativas encontradas entre los tipos de muestra, lo que permite concluir que los tres tipos de muestra difieren entre sí, presentando la EIE-R una adecuada validez de criterio discriminante.

\section{Estabilidad temporal}

Para evaluar la estabilidad temporal del instrumento, se aplicó éste en dos ocasiones espaciadas por 40 días, a una muestra de 35 participantes correspondientes a los efectivos del GOPE (Grupo de Operaciones Policiales Especiales de Carabineros) y de las Fuerzas Armadas, y participantes afectados por inundaciones. Los resultados obtenidos mediante el coeficiente producto momento de Pearson fue de $\mathrm{r}(33)=0,93, \mathrm{p}<0,001$, lo que indica que la EIE-R presenta una estabilidad temporal alta.

\section{Análisis factorial exploratorio}

Para evaluar la estructura factorial del EIE-R se llevó a cabo un análisis factorial exploratorio, empleando como método de extracción de factores el análisis de eje principal (AEP).

Para determinar el número de factores se llevó a cabo el análisis paralelo de Horn combinado con un remuestreo mediante bootstraping (muestreo con reemplazamiento) de 5.000 muestras obtenidas de la original ${ }^{17}$. El resultado obtenido de este análisis indicó que sólo el primer valor propio $(15,04)$ de la matriz de correlaciones del EIE-R alcanzó a superar al cuantil 95 de los valores propios correspondientes $(1,63)$ obtenidos a partir de las remuestras bootstraping. Este resultado lleva a concluir la existencia de sólo un factor para el EIE-R.

En cuanto a la varianza explicada por el modelo de un factor, se observa que ésta alcanzó a un $66,91 \%$. La Tabla 3 presenta la matriz de cargas factoriales para la solución de un factor.

$\mathrm{Al}$ analizar los resultados de la Tabla 3, se aprecia que la totalidad de los ítems del EIE-R presentan cargas factoriales superiores a 0,40, considerado como un valor adecuado para indicar una pertenencia significativa a un determinado factor.

Tabla 2. Validez de criterio: matriz de intercorrelaciones entre EIE-R y consumo de alcohol, síntomas ansiosos y trastornos somatomorfos

\begin{tabular}{|lccccc|}
\hline & $\mathbf{1}$ & $\mathbf{2}$ & $\mathbf{3}$ & $\mathbf{4}$ & $\mathbf{5}$ \\
1: IES-R & $(0,98)^{\mathrm{a}}$ & & & & \\
2: Depresión & $0,74^{*}$ & $(0,93)$ & & & \\
3: Alcohol & 0,10 & 0,08 & $(0,81)$ & $(0,86)$ & \\
4: Ansiedad & $0,71^{*}$ & $0,74^{*}$ & $0,18^{* *}$ & $0,77^{* *}$ & $(0,84)$ \\
\hline 5: Somatomorfo & $0,67^{*}$ & $0,63^{*}$ & 0,04 & \\
\hline
\end{tabular}

avalores entre paréntesis: Alfa de Cronbach. ${ }^{*} p<0,001$ 
Tabla 3. Cargas factoriales y comunalidad final $\left(h^{2}\right)$ de los ítems del EIE-R para la solución de un factor

\begin{tabular}{|ccc|}
\hline Ítem & Carga & $\mathbf{h}^{\mathbf{2}}$ \\
\hline 1 & 0,83 & 0,68 \\
\hline 2 & 0,79 & 0,63 \\
\hline 3 & 0,84 & 0,71 \\
\hline 5 & 0,83 & 0,69 \\
\hline 6 & 0,81 & 0,65 \\
\hline 7 & 0,87 & 0,76 \\
\hline 8 & 0,80 & 0,64 \\
\hline 9 & 0,82 & 0,67 \\
\hline 10 & 0,88 & 0,77 \\
\hline 11 & 0,86 & 0,74 \\
\hline 12 & 0,81 & 0,66 \\
\hline 13 & 0,85 & 0,73 \\
14 & 0,75 & 0,56 \\
\hline 15 & 0,90 & 0,81 \\
\hline 16 & 0,85 & 0,73 \\
\hline 17 & 0,91 & 0,84 \\
\hline 18 & 0,68 & 0,47 \\
\hline 19 & 0,86 & 0,74 \\
\hline 20 & 0,72 & 0,52 \\
\hline 21 & 0,80 & 0,65 \\
\hline 22 & 0,79 & 0,62 \\
\hline & 0,68 & 0,46 \\
\hline
\end{tabular}

\section{Discusión}

En relación a los hallazgos obtenidos en el proceso de adaptación de la EIE-R, cabe destacar que los ítems que componen la escala final son comprensibles para la población chilena, para un rango de edad comprendido entre los 16 y 64 años, con habilidades de lecto-escritura.

La EIE-R presenta alta consistencia interna y estabilidad temporal, validez convergente, al relacionarla con pruebas criterio, tales como el Inventario de Depresión de Beck y las Subescalas de Ansiedad y Trastornos Somatomorfos ${ }^{15}$, mostrando una correlación altamente significativa y una alta intensidad de la relación. Sin embargo, con el criterio Test de Identificación de Trastornos debidos al Consumo de Alcohol (AUDIT), no se encontró relación. Esto podría deberse entre otros factores, a la influencia de la deseabilidad social en el bajo reconocimiento del abuso de substancias.
La EIE-R presenta una adecuada validez discriminante, lo cual implica que ésta permite diferenciar tanto el grado de sufrimiento causado por el mismo evento en diferentes personas, así como ser sensible a variaciones en distintas poblaciones, en cuanto a intensidad del estresor.

Finalmente, con respecto a la estructura factorial, se han encontrado antecedentes de distintas soluciones en la literatura: 1, 2, 3 y hasta cuatro factores ${ }^{11}$. Al menos, nuestros hallazgos son coherentes con la adaptación realizada en China ${ }^{12}$, aunque sólo parcialmente con aquella reportada por Costa y Gracia en una adaptación preliminar en el año 2004 para el catalán ${ }^{18}$. Estos autores reportan la existencia de tres factores y una consistencia interna de 0,95.

Es importante considerar que la escala debe estar en poder de profesionales preparados y capacitados para interpretar los resultados en forma coherente y responsable, de acuerdo a los fundamentos teóricos que sustentan la escala. En esta línea, se sugiere revisar en detalle las instrucciones y descripción descritas por los autores en la publicación original (Weiss, 2004) ${ }^{7}$.

Como conclusión final, se puede afirmar que la versión chilena de la EIE-R puede ser utilizada en la población chilena, como una medición de autoreporte, con buena confiabilidad y adecuada validez para evaluar respuestas de estrés ante variados eventos traumáticos.

Agradecimientos: Agrupación de Familiares de Víctimas de Antuco, Sección GOPE Bío-Bío de Carabineros, Personal ASMAR Talcahuano, Personal Sanitario Regimiento Reforzado 17 de Los Ángeles, a la Psicóloga Maruzzella Valdivia Peralta y a todas las personas que participaron desinteresadamente en esta investigación.

\section{Referencias}

1. Carvajal C. Trastorno por estrés postraumático: aspectos clínicos. Rev Chil Neuropsiquiatr 2000; 40: 20-34.

2. Norris F. Screening for traumatic stress: A scale of use in the general population. J Appl Soc Psych 1990; 20: 1704-18.

3. Wolfe J, Kimerling R, Brown P, Chrestman K, Levin K. The Life-Stressor Checklist-Revised. En B. H. Stamm (Ed.), Instrumentation in stress, trauma, and adaptation. Northbrook, IL: SidranPress. 1996. pp. 198-200.

4. Kubany E, Haynes S, Leisen M, Owens J, Kaplan A, 
Watson S, et al. Development and preliminary validation of a brief broad-spectrum measure of trauma exposure: The Traumatic Life Events Questionnaire. Psychol Assess 2000; 12: 210-24.

5. Davidson J, Book S, Colket J, Tupler L, Roth S, David $\mathrm{D}$, et al. Assessment of a new self-rating scale for posttraumatic stress disorder. Psychol Med 1997; 27: 153-60.

6. Weathers F, Litz B, Herman D, Huska J, Keane T. The PTSD Checklist (PCL): Reliability, validity, and diagnostic utility. Paper presented at The International Society for Traumatic Stress Studies, 1993.

7. Briere, J. Trauma Symptom Inventory professional manual. Odessa, FL: Psychological Assessment Resources. 1995.

8. Wilson J, Keane, T. Assessing psychological trauma and PTSD (2 ed.). New York: Guilford Press. 2004.

9. Horowitz M, Wilner N, Álvarez W. Impact of event scale: A measure of subjective stress. Psychosom Med 1979; 41: 209-18.

10. Báguena MJ, Villarroya E, Beleña A, Díaz A, Roldán C, Reig R. Propiedades Psicométricas de la Versión Española de la escala Revisada de Impacto del Estresor. Anal Mod Cond 2001; 27: 581-604.

11. Brunet A, St-Hilaire A, Jehel L, King S. Validation of a French Version of Impact of Event Scale-Revised. Can J Psych 2003; 48: 56-61.

12. Wu K, Chan S. Psychometric Properties of the Chinese Version of Impact of Event Scale-Revised. Hong Kong J
Psych 2004; 14: 2-8.

13. Weiss D. The impact of event scale-revised. En: Wilson J \& Keane T. Editores. Assessing psychological trauma and PTSD. New York, NY: Guilford Press; 2004. p. 168-89.

14. Sanz J, Vásquez C. Fiabilidad, validez y datos normativos del Inventario para la depresión de Beck. Psicothema 1998; 10: 303-18.

15. Alvarado R, Vera A, Toledo M, Serrano F, Gatica C, Núñez C, et al. Validación de escalas para medir trastornos depresivos, por ansiedad y somatomorfes. Cuadernos de trabajo número 2. Facultad de Ciencias Humanas. Escuela de Psicología. Universidad Diego Portales. 1991.

16. Babor T, Higgins-Biddle J, Saunders J, Monteiro M. Cuestionario de identificación de los trastornos debidos al consumo de alcohol. Pautas para su utilización en atención primaria. Organización Mundial de la Salud, Departamento de Salud Mental y dependencia de sustancias. [Versión electrónica], Manual de utilización para atención primaria. 2001. MINSAL, Chile.

17. Lattin J, Carroll J, Green P. Analyzing multivariate data. Pacific Grove, CA: Thomson. 2003.

18. Costa A, De Gracia M. Evaluación del PTSD: Adaptación preliminar de las escalas IES-R y CMS. Psiquiatria. com 2004; 8. Disponible en: http://www.bibliopsiquis. com/bibliopsiquis/bitstream/10401/783/1/psiquiatriacom_2004_8_2_2.pdf [Consultado el 26 de octubre de 2010].

\section{Anexo. Ítem de Versión Chilena de Escala de Impacto de Evento-Revisada (EIE-R)}

1. Cualquier recuerdo me hacía volver a sentir lo que sentí antes

2. Tenía problemas para permanecer dormido

3. Otras cosas me hacían pensar en el suceso

4. Me sentía irritable y enojado

5. Procuraba no alterarme cuando pensaba o recordaba lo sucedido

6. Pensaba en ello aún cuando no quería

7. Sentía como si no hubiese sucedido o no fuese real

8. Me mantenía lejos de cualquier cosa que me recordara lo sucedido

9. Imágenes del suceso asaltaban mi mente

10. Me sobresaltaba y asustaba fácilmente

11. Intentaba no pensar en el suceso

12. Me daba cuenta de que quedaban muchos sentimientos sin resolver

13. Mis sentimientos sobre el suceso estaban como adormecidos

14. Me encontraba como si estuviese funcionando o sintiendo como durante el evento

15. Tenía problemas para conciliar el sueño

16. Me invadían oleadas de fuertes sentimientos sobre lo sucedido

17. Intentaba apartarlo de mi memoria

18. Tenía problemas de concentración

19. Cosas que me recordaban lo sucedido me causaban reacciones fisiológicas tales como transpiración, dificultad al respirar, náuseas o taquicardia

20. Soñaba con lo sucedido

21. Me sentía vigilante y en guardia

22. Intentaba no hablar de ello 